\title{
On the Method of Construction of the Dependence of the Heat Extension Coefficient on Temperature in Heat-resistant Alloys
}

\author{
Dr. Sairanbek Akhmetov ${ }^{1}$, Dr. Anarbay Kudaykulov ${ }^{2}$
}

\author{
${ }^{1}$ Department of Mechanics, L.N. Gumilyov Eurasian National University, Astana - 010000, Kazakhstan \\ ${ }^{2}$ Department of Fluid and Gas Mechanics, Al-Farabi Kazakh National University, Almaty - 050040, Kazakhstan
}

\begin{abstract}
In this paper, we consider methods and methods for studying rods from high-temperature alloys, in particular, the study of the dependence of the coefficient of thermal expansion on temperature. According to the tasks of the paper, methods are developed for taking into account the presence of local surface heat exchanges, temperatures, and internal point heat sources in the study of rods made of high-temperature alloys. The scientific significance of the project is due to the fact that the results of the development can be used for in-depth study of the nonlinear thermal and physical state of the structural rod elements that work in the presence of heterogeneous types of heat sources. Such designs include gas-generating, nuclear, thermal and hydrogen power plants, as well as jet engines and internal combustion engines. In order to ensure continuous reliable operation of these facilities, the authors developed fundamental methods that allow to adequately simulate nonlinear thermophysical processes in the rod bearing elements of installations taking into account simultaneous presence of local thermal insulation, heat exchange, temperatures, and internal point heat sources. The laws of the distribution of temperature, elastic, temperature and thermoelastic components of strains and stresses, as well as displacement, are obtained. This became the basis for the creation in the future of a program in the programming language PYTHON, with the help of which it is possible to construct the corresponding fields of temperature distribution, all the components of deformation, stress and displacement in the form of graphs.
\end{abstract}

Keywords-Coefficient of thermal expansion, High-temperature alloy, steady-state thermophysical process, Thermal conductivity.

\section{INTRODUCTION}

\subsection{State of the problem}

Bearing elements of many designs of power objects, such as nuclear, thermal and gas-generating power plants, as well as modern metallurgical plants, mainly work under the influence of complex heterogeneous heat sources. Some load-bearing elements of jet and hydrogen engines, as well as internal combustion engines, work similarly under the influence of local temperatures.

In most cases, the supporting elements have a structural form of a rod of limited length, and are made of heat-resistant materials. This is due to the fact that the strength characteristics of heat-resistant materials are usually higher than those of conventional materials. Simultaneous long-term exposure to rod-bearing elements of dissimilar heat sources leads to the emergence of a stable complex thermophysical state in the system. For example, in the case where the rod-bearing structural member of the structure is clamped at one end and the other end is free, it is prolonged due to the long-term action of dissimilar heat sources. In this case, the amount of elongation depends on the types and quantities of the operating heat sources, the presence of local thermal insulation and heat exchange, as well as the heat transfer coefficient, ambient temperature, rod length, thermal conductivity and thermal expansion of the heat-resistant alloy.

In the case when the rod is pinched at both ends, a steady state will occur only after the temperature distribution, a steadystate displacement distribution field and a compressive force arise, and a steady-state distribution field of the thermoelastic and temperature components of the stress and deformation appears.

To ensure reliable operation of power plants in the above situations, it is necessary to ensure the thermal strength of their load-bearing elements made of heat-resistant materials working for a long time under the influence of dissimilar kinds of heat sources. In this regard, the development of effective methods of accounting for the presence of local surface heat exchanges, temperatures, thermal insulation and internal point heat sources that arise in the structural support element in the case of manifestation of non-linear thermophysical phenomena is an actual problem. Relevance is also due to the fact that in order to obtain results of high accuracy, the process of developing methods must be based on the application of fundamental energy conservation laws for the problems under consideration. 
The solution of the above-mentioned topical problems will subsequently put forward new tasks related to the development of an application package in one of the modern programming languages where, the software products and computational algorithms of which should facilitate a series of in-depth studies.

A review of the results of previous studies by various authors showed that the disclosure of questions in the formulation discussed in this paper is insufficient.

For example, one of the studies presents the theory of finite elements for the analysis of the thermoelastic plastic reaction of solids, including the conditions for their contact [1]. In this paper, the constraint function method is used to superimpose the contact condition at the Gauss points of the contact surface. Other procedures widely used in the finite element analysis presented in this paper can be considered as special cases of the constraint function method. The solution technique presented here is promising and requires further research on the accuracy of mathematical modeling.

\subsection{Literature review}

In the author's paper [2], the main equations of thermoelasticity are given, including the laws of conservation of mass, momentum, and energy. In the results, the kinematic equations are presented, as well as the corresponding relations that close the system of equations. The numerical solution of one of the nonlinear problems of thermophysics is presented in [3]. Here we give a proof of the existence and uniqueness of the solution of the problem under consideration, the corresponding iterative scheme is applied, which makes it possible to apply the results obtained in practice.

Examples of solving the problems associated with the thermal regime of air cooling of stator windings in large turbogenerators, taking into account the possible deterioration and failure of insulation in the hole of both conventional and global YPI stators, which arise in connection with the vibration of the coil are reflected in the studies [4].

Some researchers have carried out theoretical studies on the thermoelasticity of rod elements [5]-[10].

Methods and software systems for simulating stationary thermal stress of load-bearing structural elements working under simultaneous influence of local temperatures, heat fluxes, heat transfer and thermal insulation have been developed [11]. In this work, the total dependence of the coefficient of thermal expansion on temperature is taken into account.

In some results of scientists, a functional formula is used for discretization, which indicates its total thermal energy [12], [13].

On the basis of the research, a computational algorithm and a technique for solving the problem of a given temperature field, deformations and stresses of components along the entire length of the rod were proposed [14], [15]. The work also takes into account the physical and mechanical properties of the test rod. And thermodynamic relationships, nucleation, growth and stress influence are considered and applied to thermoelasticity, pseudoelasticity and memory effects associated with martensitic transformations are presented in [16], [17].

\subsection{Problem statement and its solution}

Analysis of the state of previously conducted studies and the results obtained in them state the need for further improvement of methods and methods for constructing the dependence of the coefficient of thermal expansion on temperature in hightemperature alloys. Namely, the possibility of calculating the magnitude of the thermal elongation of the rod and the axial force arising in connection with this is not taken into account. Besides, from the results obtained by other methods one can not make an unambiguous conclusion that they really satisfy the fundamental laws of conservation of energy. To date, there has not yet been developed a fundamental mathematical model of steady nonlinear thermophysical processes in a limited length of rods made of heat-resistant alloys under simultaneous influence of local heat exchanges, temperatures, thermal insulation and internal point heat sources. In this regard, there are no corresponding computational algorithms, methods and PYTHON programs that allow simulating the established complex nonlinear processes in rods of limited length made of heat-resistant alloys under simultaneous action of dissimilar kinds of heat sources taking into account the presence of local thermal insulation. As a result, in order to solve this scientific problem, we set the following research tasks:

1) Development of methods for accounting for the presence of local surface heat exchanges in rods of a heat-resistant alloy. Development of PYTHON programs, that allows to study complex thermophysical phenomena in the core;

2) Development of a method for accounting for internal heat sources in rods of a heat-resistant alloy of limited length. Development of appropriate PYTHON programs, that allows to study the steady nonlinear thermophysical state of the investigated rod; 
3) Development of methods for the formation of composite functions characterizing the laws of conservation of energy for a limited length of a rod of a heat-resistant alloy those are under simultaneous action of local temperatures, heat exchange, thermal insulation, and internal point heat sources. Development of a software product in the programming language PYTHON, which allows investigating the arising steady nonlinear thermophysical process in the investigated rod.

4) Construction of a physico-mathematical model of the thermophysical state of a limited length of a rod made of a heatresistant alloy, which is under simultaneous action of local temperatures and thermal insulation. Development of appropriate PYTHON programs, that allows to determine the temperature field arising in the rod, thermoelastic, temperature and elastic components of deformation and stress, and also displacement. Using the software package developed on this basis, determine the value of the thermal elongation of the rod and the compressive axial force.

The above objectives of the research cover the entire research work carried out by us. The coverage of the task in full in this article was necessary to understand the issue. However, in view of the limited size of the paper, in this paper we present only the results of solving only the first problem posed. The results of solving the remaining problems will be covered in other publications of the authors.

This article presents some research results carried out by the authors within the framework of the state budget theme of the research project of the Ministry of Education and Science of the Republic of Kazakhstan, state registration number 0115RK00547, and cipher of International rubric of scientific and technical information 38.65.17.

\section{Development of The Methodology of Solving The Problem AND ITS Discussion}

2.1 Method for constructing the dependence of the coefficient of thermal expansion on temperature in hightemperature alloys

The actual experiments carried out to determine the dependence of the thermal expansion coefficient on the temperature for the sets of high-temperature alloys show that an increase in temperature leads to an increase in the coefficient of thermal expansion (see Fig. 1).

From this it can be seen that the dependence $\alpha=\alpha(T(x))$ takes place in the rods of high-temperature alloys (see Fig. 1).

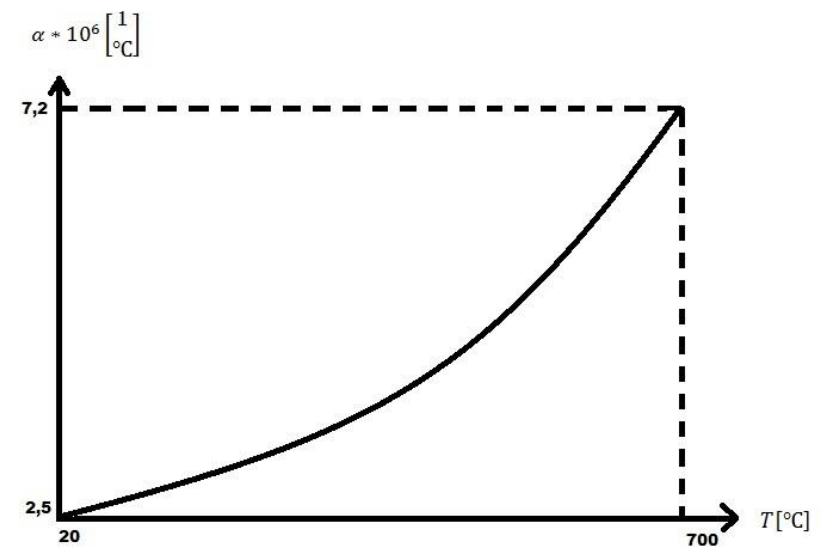

\section{Figure 1: DEPENDENCE $\alpha(T)$ FOR A High-TEMPERATURE AlLOY}

For example, consider one discrete element from a heat-resistant rod. The length element $l[\mathrm{~cm}]$ is $<<1$. The cross-sectional area of this element will be denoted by $S\left[\mathrm{~cm}^{2}\right]$. In this case, it is constant along the length of the element. Through the area of the lateral surface of this element, heat exchange takes place with its surrounding medium. Heat transfer coefficient is denoted by $h\left[\mathrm{~W} / \mathrm{cm}^{2 \circ} \mathrm{C}\right]$. Ambient temperature is $T_{\text {amb }}\left[{ }^{\circ} \mathrm{C}\right]$. The physico-mechanical property of the core material from the heat-resistant alloy is characterized by the dependence of the coefficient of thermal expansion $\alpha$ on the temperature distribution field, $T(x)$ i.e. $\alpha=\alpha(T(x))$. Hence, the coefficient of thermal expansion of material $\alpha$ will also depend on the coordinate $x$.

In addition, the physical and mechanical properties of the rod material are characterized by the coefficient of thermal conductivity of the material of the rod $k_{x}\left[\frac{\mathrm{W}}{\mathrm{cm}^{2{ }^{\circ} \mathrm{C}}}\right]$ and the elastic modulus $E\left[\frac{\mathrm{kg}}{\mathrm{cm}^{2}}\right]$. Taking into account that the length of the considered discrete element is much smaller $(0.1-1) \mathrm{mm}$, then the field of temperature distribution and coefficient of 
thermal expansion along the length of this element is approximated by a complete second-order polynomial. The calculation scheme of the problem under study is shown in Fig. 2.

We introduce the following notation:

$$
T(x=0)=T_{i} ; \quad T\left(x=\frac{l}{2}\right)=T_{j} ; \quad T(x=l)=T_{k}
$$

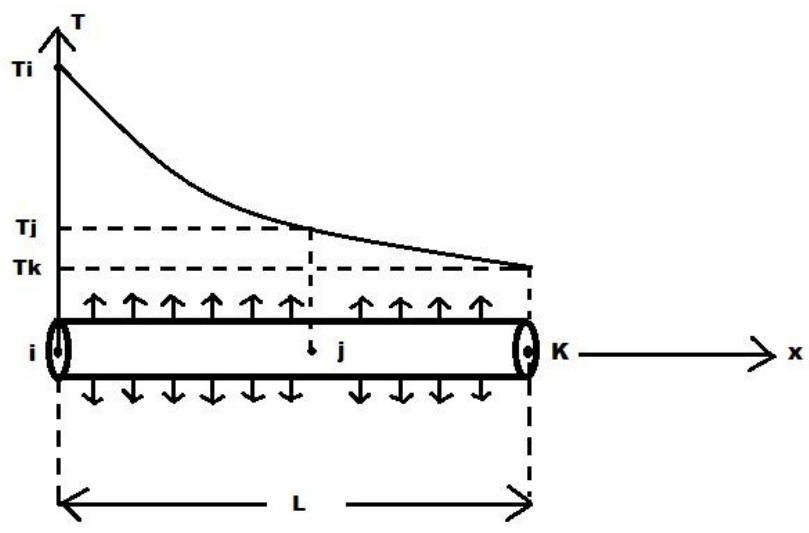

(A) Change in TEMPerature AlONG THE LENGTH OF THE ROD

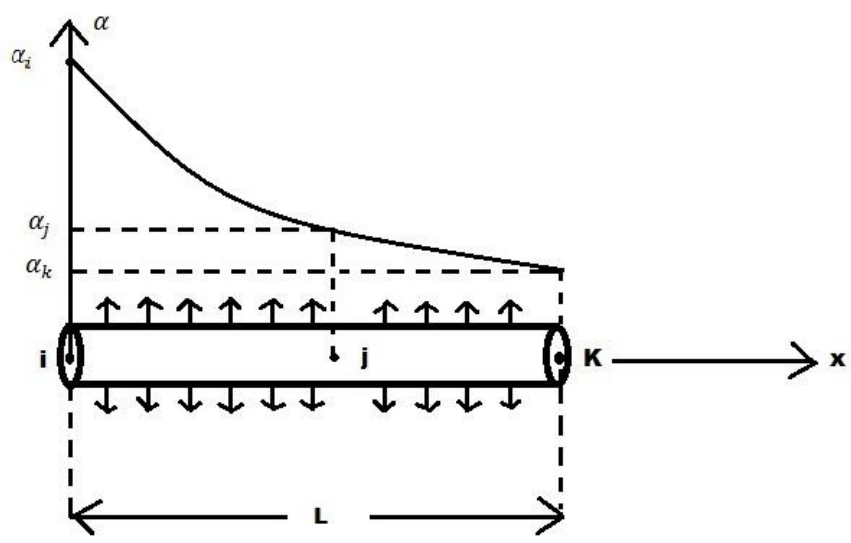

(B) CHANGE OF THE GENERAL FUNCTION FOR NODAL ELEMENTS

\section{Figure 2: CAlCUlation Scheme OF THE Discrete ElEMENT UNDER CONSIDERATION}

The temperature field distribution along the length of one discrete element is approximated by a complete polynomial of the second order:

$$
T(x)=a x^{2}+b x+c, 0 \leq x \leq l ; \quad a, b, c=\text { const }
$$

Here, the values of the constants $a, b$ and $c$ are unknown yet. Such an approximation of the temperature field is based on the fact that the process under consideration is a steady-state thermal conductivity. In this connection, in a small section $(0 \leq$ $\mathrm{x} \leq \mathrm{l}<<<\mathrm{L}$ ) of the rod, the law of temperature distribution can be approximated by a curve of the second order. Then from (1) we obtain the following system of linear algebraic equations for determining the values of the constants $a, b$ and $c$ :

$$
\begin{aligned}
& T(x=0)=a \cdot 0+b \cdot 0+c=T_{i} \\
& T\left(x=\frac{l}{2}\right)=a \cdot\left(\frac{l}{2}\right)^{2}+b \cdot \frac{l}{2}+c=T_{j} \\
& T(x=l)=a \cdot l^{2}+b \cdot l+c=T_{k}
\end{aligned}
$$

Hence from the first equation we have that:

$$
c=T_{i} .
$$

Further, from the last two equations we obtain:

$$
\begin{aligned}
& a l^{2}+2 b l=4 T_{j}-4 T_{i} \\
& a l^{2}+b l=T_{k}-T_{i}
\end{aligned}
$$

By subtracting the second equation from the first, we obtain:

$$
\begin{aligned}
& b l=4 T_{j}-4 T_{i}-T_{k}+T_{i}=4 T_{j}-3 T_{i}-T_{k} \\
& b=\frac{4 T_{j}-3 T_{i}-T_{k}}{l}
\end{aligned}
$$

Substituting (5) into the second equation of system (4), we get:

$$
a l^{2}+\frac{4 T_{j}-3 T_{i}-T_{k}}{l} l=T_{k}-T_{i}
$$




$$
\begin{aligned}
& a l^{2}=T_{k}-T_{i}-4 T_{j}+3 T_{i}+T_{k}=2 T_{k}+2 T_{i}-4 T_{j} \\
& a=\frac{2 T_{k}+2 T_{i}-4 T_{j}}{l^{2}}
\end{aligned}
$$

Now, substituting the values of $\mathrm{a}, \mathrm{b}$ and $\mathrm{c}$ in equation (1) we obtain:

$$
T(x)=\left(\frac{2 T_{k}+2 T_{i}-4 T_{j}}{l^{2}}\right) x^{2}+\frac{4 T_{j}-3 T_{i}-T_{k}}{l} x+T_{i}, 0 \leq x \leq l
$$

We rewrite the last expression in the following form:

$$
T(x)=(\ldots) T_{i}+(\ldots) T_{j}+(\ldots) T_{k}
$$

i.e.

$$
T(x)=\left(\frac{2 x^{2}}{l^{2}}-\frac{3 x}{l}+1\right) T_{i}+\left(\frac{4 x}{l}-\frac{4 x^{2}}{l^{2}}\right) T_{j}+\left(\frac{2 x^{2}}{l^{2}}-\frac{x}{l}\right) T_{k}
$$

or

$$
T(x)=\left(\frac{2 x^{2}-3 l x+l^{2}}{l^{2}}\right) T_{i}+\left(\frac{4 l x-4 x^{2}}{l^{2}}\right) T_{j}+\left(\frac{2 x^{2}-l x}{l^{2}}\right) T_{k}, \quad 0 \leq x \leq l
$$

We introduce the following notation:

$$
\varphi_{i}(x)=\frac{2 x^{2}-3 l x+l^{2}}{l^{2}} ; \varphi_{j}(x)=\frac{4 l x-4 x^{2}}{l^{2}} ; \varphi_{k}(x)=\frac{2 x^{2}-l x}{l^{2}}, 0 \leq x \leq l
$$

These functions are called form functions for three nodes of a quadratic discrete element in the local coordinate system $0 \leq \mathrm{x} \leq \mathrm{l}$. These functions have the following properties:

$$
\begin{gathered}
\varphi_{i}(x)= \begin{cases}\text { when } x=0, & \varphi_{i}(0)=1 ; \\
\text { when } x=\frac{l}{2}, & \varphi_{i}\left(\frac{l}{2}\right)=0 ; \\
\text { when } x=l, & \varphi_{i}(0)=0 ;\end{cases} \\
\varphi_{j}(x)= \begin{cases}\text { when } x=0, & \varphi_{j}(0)=0 ; \\
\text { when } x=\frac{l}{2}, & \varphi_{j}\left(\frac{l}{2}\right)=1 ; \\
\text { when } x=l, & \varphi_{j}(0)=0 ;\end{cases} \\
\varphi_{k}(x)= \begin{cases}\text { when } x=0, & \varphi_{k}(0)=0 ; \\
\text { when } x=\frac{l}{2}, & \varphi_{k}\left(\frac{l}{2}\right)=0 ; \\
\text { when } x=l, & \varphi_{k}(0)=1\end{cases}
\end{gathered}
$$

Also these functions have the following properties:

$$
\varphi_{i}(x)+\varphi_{j}(x)+\varphi_{k}(x)=\frac{2 x^{2}-3 l x+l^{2}+4 l x-4 x^{2}+2 x^{2}-l x}{l^{2}}=1
$$

These properties of the form function make it possible to provide continuity conditions for the desired function in the transition from one discrete element to another.

From (7) it is also possible to determine the temperature gradient in the local coordinate system

$$
\frac{\partial T}{\partial x}=\frac{\partial \varphi_{i}}{\partial x} T_{i}+\frac{\partial \varphi_{j}}{\partial x} T_{j}+\frac{\partial \varphi_{k}}{\partial x} T_{k}=\left(\frac{4 x-3 l}{l^{2}}\right) T_{i}+\left(\frac{4 l-8 x}{l^{2}}\right) T_{j}+\left(\frac{2 x-l}{l^{2}}\right) T_{k}, 0 \leq x \leq l
$$

Similarly to (7) within the length of one discrete element, the distribution field of the thermal distribution coefficient is also approximated by a curve of the second order:

$$
\alpha(T(x))=\varphi_{i}(x) \cdot \alpha_{i}+\varphi_{j}(x) \cdot \alpha_{j}+\varphi_{k}(x) \cdot \alpha_{k}, 0 \leq x \leq l
$$


Here $\alpha_{i}\left(T_{i}\right), \alpha_{j}\left(T_{j}\right)$ и $\alpha_{k}\left(T_{k}\right)$ are determined from the directory. Dependence $\alpha=\alpha(T)$ is determined for each heat resistant material separately.

\subsection{The method of accounting for the presence of local surface heat exchanges in rods of high-temperature alloys}

Consider one discrete element of the rod through the lateral surface of which there is a heat exchange with its surrounding medium (see Fig. 3).

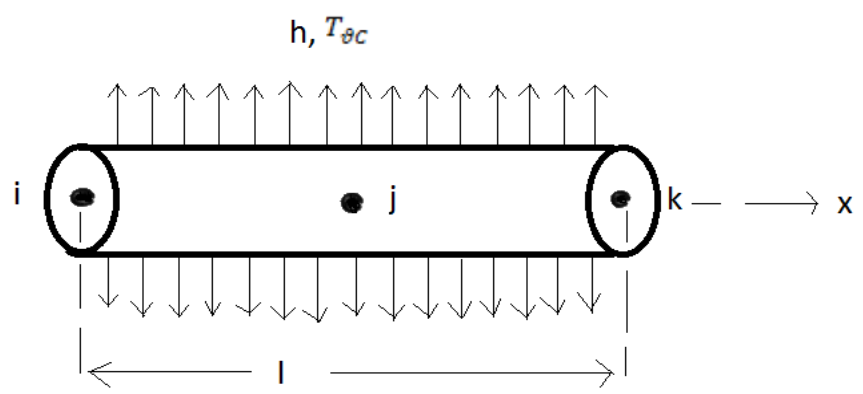

\section{Figure 3: Calculation Scheme for the Problem of Local Surface Heat Exchange}

Heat transfer coefficient $h\left[\frac{W}{\mathrm{~cm}^{2 .}{ }^{\circ} \mathrm{C}}\right]$, ambient temperature $T_{a m b}\left[{ }^{\circ} \mathrm{C}\right]$. For the problem under consideration, we write the expression for the functional that characterizes the law of conservation of energy:

$$
J=\int_{V} \frac{k_{x x}}{2}\left(\frac{\partial T}{\partial x}\right)^{2} d V+\int \frac{h}{2}\left(T-T_{o c}\right)^{2} d S, \quad 0 \leq x \leq l
$$

Where $\mathrm{V}$ is the volume of the discrete element: $S_{l s}$ - is the area of the lateral surface of the discrete element under consideration.

The unit of measurement of the first member will be:

$$
\frac{W}{\mathrm{~cm} \cdot{ }^{\circ} \mathrm{C}} \cdot \frac{{ }^{\circ} \mathrm{C}^{3}}{\mathrm{~cm}^{2} \cdot{ }^{\circ} \mathrm{C}} \cdot \mathrm{cm}^{3}=W \cdot{ }^{\circ} \mathrm{C}
$$

The unit of measurement of the second term will also be:

$$
\frac{W}{\mathrm{~cm}^{2 .}{ }^{\circ} \mathrm{C}} \cdot{ }^{\circ} \mathrm{C}^{2} \cdot \mathrm{cm}^{2}=W \cdot{ }^{\circ} \mathrm{C}
$$

We now integrate the first term in (13):

$$
J_{1}=\int_{V} \frac{k_{x x}}{2}\left(\frac{\partial T}{\partial x}\right)^{2} d V=F \int_{0}^{l} \frac{k_{x x}}{2}\left(\frac{\partial T}{\partial x}\right)^{2} d V=\frac{F \cdot k_{x}}{2} \int_{0}^{l} \frac{k_{x x}}{2}\left(\frac{\partial T}{\partial x}\right)^{2} d V ;
$$

Here, $F\left[\mathrm{~cm}^{2}\right]$ is the cross-sectional area of the rod. It is constant along the entire length of the rod. In addition, the crosssection of the rod can be a circle, an ellipse, and any polygon. Further substituting the formula (1) in the last expression of $J_{1}$, we obtain:

$$
\begin{gathered}
J_{1}=\frac{F k_{x}}{2} \int_{0}^{l}\left[\left(\frac{4 x-3 l}{l^{2}}\right) T_{i}+\left(\frac{4 l-8 x}{l^{2}}\right) T_{j}+\left(\frac{4 x-l}{l^{2}}\right) T_{k}\right]^{2} d x=\frac{F k_{x x}}{2 l^{4}} \int_{0}^{l}\left[\left(16 x^{2}-24 l x+9 l^{2}\right) T_{i}^{2}+2\left(40 l x-32 x^{2}-12 l^{2}\right) T_{i} T_{j}+\right. \\
\left.+2\left(16 x^{2}-16 l x+3 l^{2}\right) T_{i} T_{k}+\left(16 l^{2}-64 l x+64 x^{2}\right) T_{j}^{2}+2\left(24 l x-4 l^{2}-32 x^{2}\right) T_{j} T_{k}+\left(16 x^{2}-8 l x+l^{2}\right) T_{k}^{2}\right] d x= \\
\quad=\frac{F k_{x x}}{2 l^{4}} \int_{0}^{l}\left[\left(\frac{16 x^{3}}{3}-12 l x^{2}+9 l^{2} x\right) T_{i}^{2}+2\left(40 l x^{2}-\frac{64 x^{3}}{3}-24 l^{2} x\right) T_{i} T_{j}+\left(\frac{32 x^{3}}{3}-16 l x^{2}+6 l^{2} x\right) T_{i} T_{k}+\right. \\
\left.\quad+\left(16 l^{2}-32 l x+\frac{64 x^{3}}{3}\right) T_{j}^{2}+\left(24 l x^{2}-81 l^{2} x-\frac{64 x^{3}}{3}\right) T_{j} T_{k}+\left(\frac{16 x^{3}}{3}-4 l x^{2}+l^{2} x\right) T_{k}^{2}\right] d x= \\
=\frac{F k_{x x} l^{3}}{2 l^{4}}\left[\frac{7}{3} T_{i}^{2}-\frac{16}{3} T_{i} T_{j}+\frac{2}{3} T_{i} T_{k}+\frac{16}{3} T_{j}^{2}-\frac{16}{3} T_{j} T_{k}+\frac{7}{3} T_{k}^{2}\right]=\frac{F k_{x x}}{6 l}\left(7 T_{i}^{2}-16 T_{i} T_{j}+2 T_{i} T_{k}+16 T_{j}^{2}-16 T_{i} T_{k}+7 T_{k}^{2}\right)
\end{gathered}
$$

Here it should be noted that the sum of the coefficients before the node temperatures will always be zero. For example, in our case $(7-16+2+16-16+7)=0$. This is a sign of the fulfillment of the law of conservation of energy.

We now integrate the second term in (13): 


$$
J_{2}=\int_{S_{l s}} \frac{h}{2}\left(T-T_{a m b}\right)^{2} d s=P \int_{0}^{l} \frac{h}{2}\left(T-T_{a m b}\right)^{2} d x .
$$

Here $\mathrm{P}$ is the perimeter of the cross-section of the rod. Substituting (7) in the expression $J_{2}$, we obtain:

$$
\begin{gathered}
J_{2}=\frac{P h}{2} \int_{0}^{l}\left[\left(\frac{2 x^{2}-3 l x+l^{2}}{l^{2}}\right) T_{i}+\left(\frac{4 l x-4 x^{2}}{l^{2}}\right) T_{j}+\left(\frac{2 x^{2}-l x}{l^{2}}\right) T_{k}-T_{a m b}\right]^{2} d x= \\
=\frac{P h}{2} \int_{0}^{l}\left[\left(\frac{4 x^{4}-12 l x^{3}+13 l^{2} x^{2}-6 l^{3}+l^{4}}{l^{4}}\right) T_{i}^{2}+\left[\left(\frac{40 l x^{3}-16 x^{4}-32 l^{2} x^{2}-8 l^{3} x}{l^{4}}\right) T_{i} T_{j}++\left(\frac{8 x^{4}-40 l x^{3}+10 l^{2} x^{2}-2 l^{3} x}{l^{4}}\right) T_{i} T_{k}+\right.\right. \\
+\left(\frac{16 l^{2} x^{2}-32 l x^{3}+16 x^{4}}{l^{4}}\right) T_{j}^{2}+\left(\frac{24 l x^{3}-8 l^{2} x^{2}-16 x^{4}}{l^{4}}\right) T_{i} T_{k}+\left(\frac{16 x^{4}-32 l x^{3}+l^{2} x^{2}}{l^{4}}\right) T_{k}^{2}- \\
\left.-\left(\frac{4 x^{2}-6 l x+2 l^{2}}{l^{4}}\right) T_{a m b} T_{i}-\left(\frac{8 l x-8 x^{2}}{l^{4}}\right) T_{a m b} T_{j}-\left(\frac{4 x^{2}-2 l x}{l^{4}}\right) T_{a m b} T_{k}+T_{a m b}^{2}\right] d x= \\
=\frac{P h l}{30}\left(2 T_{j}^{2}+2 T_{i} T_{j}-T_{i} T_{k}+8 T_{j}^{2}+2 T_{k}^{2}+2 T_{j} T_{k}-5 T_{a m b} T_{i}-20 T_{a m b} T_{j}-5 T_{a m b} T_{k}+15 T_{a m b}^{2}\right)
\end{gathered}
$$

It is also seen here that the sum of the coefficients before the temperatures will be zero. In our case $(2+2-1+8+2+2-5-20-$ $5+15)=0$. This is a sign of the fulfillment of the law of conservation of energy. Then substituting (14) and (15) into (13) we obtain an integrated form of the functions $J$ - which characterizes the law of conservation of energy for the discrete element under investigation, through whose lateral surface heat exchange takes place with its surrounding medium:

$$
\begin{aligned}
& J=J_{1}+J_{2}=\frac{F k_{x x}}{6 l}\left(7 T_{i}^{2}-16 T_{i} T_{j}+2 T_{i} T_{k}+16 T_{j}^{2}-16 T_{i} T_{k}+7 T_{k}^{2}\right)+\frac{P h l}{30}\left(2 T_{j}^{2}+2 T_{i} T_{j}-T_{i} T_{k}+\right. \\
& \left.+8 T_{j}^{2}+2 T_{k}^{2}+2 T_{j} T_{k}-5 T_{a m b} T_{i}-20 T_{a m b} T_{j}-5 T_{a m b} T_{k}+15 T_{a m b}^{2}\right)
\end{aligned}
$$

Further minimizing $\mathrm{J}$ from the desired $T_{i}, T_{j}$ and $T_{k}$ we obtain a resolving system of linear algebraic equations taking into account the presence of local lateral heat exchange:

$$
\begin{aligned}
& \frac{\partial J}{\partial T_{i}}=0 ;=>\frac{F k_{x x}}{6 l}\left(14 T_{i}-16 T_{j}+2 T_{k}\right)+\frac{P h l}{30}\left(4 T_{i}+2 T_{j}-T_{k}-5 T_{a m b}\right)=0 \\
& \frac{\partial J}{\partial T_{j}}=0 ;=>\frac{F k_{x x}}{6 l}\left(-16 T_{i}-16 T_{k}+32 T_{j}\right)+\frac{P h l}{30}\left(2 T_{i}+16 T_{j}+2 T_{k}-20 T_{a m b}\right)=0 \\
& \frac{\partial J}{\partial k}=0 ;=>\frac{F k_{x x}}{6 l}\left(2 T_{i}-16 T_{j}+14 T_{k}\right)+\frac{P h l}{30}\left(-T_{i}+2 T_{j}+4 T_{k}-5 T_{a m b}\right)=0
\end{aligned}
$$

Here it was meant that the cross-sectional areas of the two ends of the discrete element of the rod are thermally insulated. Solving the last system, we find the nodal temperatures:

$$
\begin{aligned}
& T_{k}=\frac{\left[2 b_{1}\left(a_{11} a_{22}-a_{12} a_{21}\right)-b_{1}\left(3 a_{11}-2 a_{21}\right) a_{12}\right]}{2\left[\left(a_{11}+a_{13}\right)\left(a_{11} a_{22}-a_{12} a_{21}\right)-a_{12} a_{21}\left(a_{11}-a_{13}\right)\right]} ; \\
& T_{j}=\frac{\left(a_{13} a_{21}-a_{11} a_{21}\right)}{\left(a_{11} a_{22}-a_{12} a_{21}\right)} T_{k}+\frac{b_{1}\left(3 a_{11}-2 a_{21}\right)}{2\left(a_{11} a_{22}-a_{12} a_{21}\right)} ; \\
& T_{i}=-4 T_{j}+15 T_{k}-400 ; \\
& a_{11}=\left(7+\frac{4 h l^{2}}{5 k_{x}}\right) ; a_{12}=\left(\frac{2 h l^{2}}{5 k_{x}}-8\right) ; a_{13}=\left(1-\frac{h l^{2}}{5 k_{x}}\right) ; a_{11}=\left(7+\frac{4 h l^{2}}{5 k_{x}}\right) ; \\
& b_{1}=\frac{h l^{2} T_{o c}}{k_{x}} ; a_{21}=\left(1+\frac{3 h l^{2}}{20 k_{x}}\right) ; a_{22}=\left(2+\frac{6 h l^{2}}{5 k_{x}}\right) ; \\
& T(x)=\varphi_{i}(x) T_{i}+\varphi_{j}(x) T_{j}+\varphi_{k}(x) T_{k}=\frac{2 x^{2}-3 l x+l^{2}}{l^{2}} T_{i}+\frac{4 l x-2 x^{2}}{l^{2}} T_{j}+\frac{2 x^{2}-l x}{l^{2}} T_{k}
\end{aligned}
$$

If we take $r=1 \mathrm{~cm}$ for the initial data; $F=\pi r^{2}=\pi \mathrm{cm}^{2} ; P=2 \pi r=2 \pi \mathrm{cm} ; k_{x x}=100\left[\frac{\mathrm{W}}{\mathrm{cm}^{\circ} \mathrm{C}}\right] ; l=10 \mathrm{~cm}$; $h=10\left[\frac{\mathrm{W}}{\mathrm{cm}^{20} \mathrm{C}}\right]$, it follows from (17) we find that:

$$
T_{i}=T_{k}=37,037^{\circ} \mathrm{C} ; T_{j}=29.62963^{\circ} \mathrm{C}
$$


Then the law of temperature distribution along the length of the investigated rod will be as follows:

$$
\begin{aligned}
& T(x)=\varphi_{i}(x) T_{i}+\varphi_{j}(x) T_{j}+\varphi_{k}(x) T_{k}= \\
& =\frac{1}{12}\left[\left(2 x^{2}-3 x l+l^{2}\right) T_{i}+(4 x l-4 x) T_{j}+\left(2 x^{2}-x l\right) T_{k}\right]= \\
& =\frac{1}{100}\left[\left(2 x^{2}-30 x+100\right) \cdot 37.037+\left(40 x-4 x^{2}\right) \cdot 29.62963+\left(2 x^{2}-10 x\right) \cdot 37.037\right]= \\
& =\frac{1}{100}\left[(148.148-118.51852) x^{2}+(1185.1852-1111.11-370.37) x+3703.7\right]= \\
& =\frac{1}{100}\left[29.62948 x^{2}+296.2948 x+3703.7\right]=0.2962948 x^{2}-2.962948 x+37.037 ; 0 \leq x \leq l
\end{aligned}
$$

Here, because of the symmetry of the problem under study $T_{i}=T_{k}$. Further substituting (17) into (7) we find the law of temperature distribution. If the left end of the test element is rigid, that it lengthens. According to the fundamental laws of thermophysics, the magnitude of the elongation of the rod element is determined by the formula $\Delta l_{T}=\int_{0}^{l} \alpha T(x) d x$. Here it must be taken into account that the values of the coefficient of warm expansion of the material of rod $\alpha$ for different heatresistant materials will be different. They are determined experimentally for different temperatures and materials. If we take $\alpha=$ const, then we get that:

$$
\begin{aligned}
& \Delta l_{T}=\alpha \int_{0}^{l}\left[\varphi_{i}(x) T_{i}+\varphi_{j}(x) T_{j}+\varphi_{k}(x) T_{k}\right] d x=\alpha \int_{0}^{l}\left[\frac{2 x^{2}-3 l x+l^{2}}{l^{2}} T_{i}+\frac{4 l x-4 x^{2}}{l^{2}} T_{j}+\frac{2 x^{2}-l x}{l^{2}} T_{j}\right] d x= \\
& =\left.\frac{\alpha}{l^{2}}\left[\left(\frac{2 x^{3}}{3}-\frac{3 l x^{2}}{2}+l^{2} x\right) T_{i}+\left(2 l^{2} x-\frac{4 x^{3}}{3}\right) T_{j}+\left(\frac{2 x^{3}}{3}-\frac{l x^{2}}{2}\right) T_{k}\right]\right|_{0} ^{l}=\alpha l\left[\left(\frac{4-9+6}{6}\right) T_{i}+\left(\frac{6-4}{3}\right) T_{j}+\left(\frac{4-3}{6}\right) T_{k}\right]= \\
& =\alpha l\left[\frac{1}{6} T_{i}+\frac{4}{6} T_{j}+\frac{1}{6} T_{k}\right]=\frac{\alpha l}{6}\left(T_{i}+4 T_{j}+T_{k}\right)
\end{aligned}
$$

If both ends of the rod are rigidly clamped, an axial compressive force occurs in the rod (see Fig. 4):

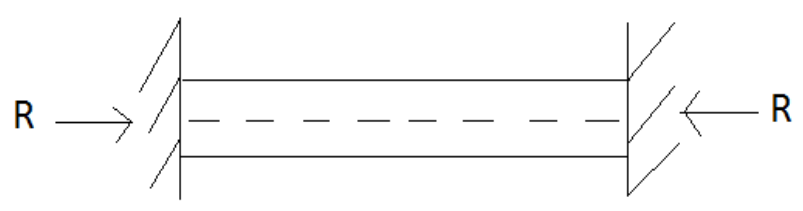

\section{FIGURE 4: EFFECT OF COMPRESSIVE FORCES}

The value of this force is determined from the compatibility condition of the deformation:

$$
\frac{R l}{E F}+\Delta l_{T}=0=>R=-\frac{\Delta l_{T} E F}{l}=-\frac{E F l}{6}
$$

In this case, a thermoelastic stress component arises in the cross sections of the rod. It is determined in accordance with Hooke's law:

$$
\sigma=E \varepsilon=>\varepsilon=\frac{\sigma}{E}=-\frac{\alpha}{6}\left(T_{i}+4 j+T_{k}\right), 0 \leq x \leq l
$$

It should be noted that in the rods of heat-resistant materials, in the case of pinching the two ends and conventional heat transfer through the side surface, the temperature and elastic components of the strain and stress also appear. For example, the field of distribution of the temperature component of the deformation is determined in accordance with the fundamental laws of thermal physics:

$$
E_{T}(x)=-\alpha T(x)=-\frac{\alpha}{l^{2}}\left[\left(2 x^{2}-3 l x+l^{2}\right) T_{i}+\left(4 l x-4 x^{2}\right) T_{j}+\left(2 x^{2}-l x\right) T_{k}\right], \quad 0 \leq x \leq l
$$

Then, in accordance with the generalized Hooke's law, the distribution of the temperature component voltage is determined by the formula:

$$
\sigma_{T}(x)=E \varepsilon_{T}(x)=-\frac{\alpha}{l^{2}}\left[\left(2 x^{2}-3 l x+l^{2}\right) T_{i}+\left(4 l x-4 x^{2}\right) T_{j}+\left(2 x^{2}-l x\right) T_{k}\right], \quad 0 \leq x \leq l
$$

In addition to these components, the field of distribution of the elastic deformation component also takes place in the investigated rod. It is determined from the following relation: 


$$
\begin{aligned}
& \varepsilon=E_{x}(x)+\varepsilon_{T}(x)=>\varepsilon_{x}(x)=\varepsilon-\varepsilon_{T}(x)=-\frac{\alpha}{6}\left(T_{i}+4 j+T_{k}\right)+ \\
& +\frac{\alpha}{l^{2}}\left[\left(2 x^{2}-3 l x+l^{2}\right) T_{i}+\left(4 l x-4 x^{2}\right) T_{j}+\left(2 x^{2}-l x\right) T_{k}\right], 0 \leq x \leq l
\end{aligned}
$$

The corresponding field of distribution of the elastic component of the voltage is determined in accordance with the generalized Hooke's law:

$$
\begin{aligned}
& \sigma_{x}(x)=\sigma-\sigma_{T}(x)=E \varepsilon_{x}(x)=-\frac{\alpha E}{6}\left(T_{i}+4 j+T_{k}\right)+ \\
& +\frac{\alpha E}{l^{2}}\left[\left(2 x^{2}-3 l x+l^{2}\right) T_{i}+\left(4 l x-4 x^{2}\right) T_{j}+\left(2 x^{2}-l x\right) T_{k}\right], 0 \leq x \leq l
\end{aligned}
$$

Finally, we can determine the law of distribution of displacement along the length of the rod. It is determined from the general Cauchy relations:

$$
\begin{aligned}
& \varepsilon_{x}(x)=\frac{\partial U}{\partial x} ;=>U(x)=\int \varepsilon_{x}(x) d x=-\frac{\alpha}{6}\left(T_{i}+4 j+T_{k}\right) x+ \\
& +\frac{\alpha}{l^{2}}\left[\left(\frac{2 x^{3}}{3}-\frac{3 l x^{2}}{2}+l^{2} x\right) T_{i}+\left(2 l x^{2}-\frac{4 x^{3}}{3}\right) T_{j}+\left(\frac{2 x^{3}}{3}-\frac{l x^{2}}{2}\right) T_{k}\right]+C
\end{aligned}
$$

Here $\mathrm{C}$ is the integration constant. Its values are determined from the boundary condition at $\mathrm{x}=0$. Since this end of the investigated rod is rigidly constrained, then for $x=0, U(x=0)=0$. From this physical condition we get that $C=0$. Then the law of distribution of displacement along the length of the rod will have the following form:

$$
U(x)=-\frac{\alpha}{6}\left(T_{i}+4 j+T_{k}\right) x+\frac{\alpha}{6 l^{2}}\left[\left(6 l^{2} x\right) T_{i}+\left(12 l x^{2}-8 x^{3}\right) T_{j}+\left(4 x^{3}-3 l x^{2}\right) T_{k}\right], 0 \leq x \leq l
$$

\section{CONCLUSION}

The developed technique for taking into account the simultaneous presence in the rod of the heat-resistant alloy of local surface heat exchanges allowed:

1) To obtain a resolving system of linear algebraic equations taking into account natural boundary conditions for a limited length of a rod made of a heat resistant alloy under the influence of local surface heat exchange;

2) To obtain, within the framework of the task at hand, the laws for the distribution of temperature, elastic, temperature, and thermoelastic components of deformations and stresses, and also for displacement;

3) Within the framework of the task set, to create a program in PYTHON programming language, with the help of which the corresponding fields of temperature distribution, all the components of deformation, stress and displacement in the form of graphs are constructed.

\section{REFERENCES}

[1] J. Deang, Q. Du, and M. Gunzburger, "Modeling and computation of random thermal fluctuations and material defects in the Ginzburg-Landau model for superconductivity", J. Computational Physics, vol. 181, pp. 45-67, 2002

[2] B. A. Biswajit, "Material Point Method Formulation for Plasticity", J. Computational Physics, vol. 51, pp. 1-25, 2006.

[3] D. Pantusoa, Klaus-Juergen Bathe, P. A. Bouzinov, "A finite element procedure for the analysis of thermo-mechanical solids in contact", Computers and Structures 75, vol. 2. pp. 551-573, 2000.

[4] B. Z. Kenzhegul, A. K. Kudaykulov, and A. N. Myrzasheva, "Numerical study of stem elongation of heat-resistant alloy based on the availability of all types of sources", J. Science and new technologies, vol. 3. pp. 67-75, 2009.

[5] M. D. Greenberg, J. Pryor, and W. Elban, "On the Formulation of the Zero Creep Method for Small Diameter Wires", Materials Science and Engineering, vol. 33. pp. 63-67, 1978.

[6] M. E. Gurtin, "An Introduction to Continuum Mechanics", New York: Academic Press, 830 p., 1981.

[7] O. A. Maugin, "The Thermomechanics of Nonlinear Irreversible Behaviors: An Introduction", Singapore: World Scientific, 375 p, 1999.

[8] T. Belytschko, У. Guo, W. Liu, and S. Xiao, "A unified stability analysis of meshless particle methods", Int. J. Numerical Methods in Engineering, no. 48 (9), pp. 1359-1400, 2000.

[9] T. W. Wright, "The Physics and Mathematics of Adiabatic Shear Bands", Cambridge: Cambridge University Press, UK, 240 p., 2002.

[10] R. C. Batra, "Elements of Continuum Mechanics", American Institute of Aeronautics and Astronautics, 325 p., 2006.

[11] Zh. M. Tashenova, E. N. Nurlybaev, and A. K. Kudaiykulov, "Method of Solution and Computational Algorithm for Mixed ThermoMechanics Problem", World Applied Sciences J., (Spec. Issue on Techniq. and Technol., vol. 22, pp. 49-57, 2013. 
[12] K. H. Huebner, "The Finite Element Method for Engineers", Wiley, pp. 183- 187, 1975.

[13] Lishirong Yangjingning, "Accurate model of post bucking of elastic rod with Mirabel cross sections", Gansu University of Science, vol. 1, pp. 98-102, 1999.

[14] Zh. M. Tashenova, E. Nurlybaeva, and A. K. Kudaykulov, "Method Preparation and Solution Algorithm for Resolving Stationary Problem of a Rod under Thermo-Stressed Condition Restrained at both Ends Affected by Heat Exchange and Heat Flows", World Applied Sciences J., (Spec. Issue on Techniq. and Technol.), vol. 22, pp. 49-57, 2013.

[15] Wu Jie, Wei Dong Yang, and Yu. ZhiHao, "An Examination of Blade Load Ca1culations Based oп Rigid-Flexible Coupling Model" J. Applied Mechanics and Materials, vol. 18, pp. 741- 745, 2013.

[16] L. Delaey, R. V. Krishnam, and H. Tas, Warlimont, "Thermoelasticity, pseudoelasticity and the memory effects associated with martensitic transformations", J. of Materials Science, no. 9, pp. 1359 -1363, 1974.

[17] R. J. Wasilewski, "The effect of applied stress оп the martensitic transformations in TiNi", J. Met. Trans, vol. 2, no. 11, pp. 29732981, 1975.

\section{AUThor Details}

Dr. Sairanbek Akhmetov is a Professor of the L.N. Gumilyov Eurasian National University, who made researches in mechanisms and machinery dynamics; moreover, he is the editor-in-chief and chairman of the editorial board of several Russian and Kazakh scientific journals, an acting member of the National Academy of the Republic of Kazakhstan and of the Russian Academy of Natural Sciences. He published more than 200 scientific papers, prepared $18 \mathrm{PhD}$ in technical direction.

Dr. Anarbay Kudaykulov is a Professor of Al-Farabi Kazakh National University, who is actively involved in reading special courses in mechanics, fluid and gas in the leading universities of Russia and China, whose scientific interests include dynamic and thermal processes in liquid medium and in structural elements of machines and mechanisms. He is the author of more than 300 published papers. 